\title{
Meshing principle analysis of 3-DOF involute spherical gear
}

\author{
Baichao Wang ${ }^{1}$, Xue Zhang ${ }^{1}$, Litong Zhang ${ }^{1 *}$ and Xianting Lu ${ }^{1}$ \\ ${ }^{1}$ Mechanical and Electrical Engineering, Changchun University Of Science And Technology, Changchun, Jilin, 130022, China
}

\begin{abstract}
In this paper, a mathematical model of meshing motion of three degree of freedom involute spherical gear pair is constructed. The mathematical model can realize continuous meshing transmission between gear pairs without transmission principle error. Based on the meshing principle and motion analysis of the gear, the tooth profile of the spherical gear is designed by combining the two tooth surfaces of the involute ring gear and the hemispherical bevel gear. According to the conjugate motion relationship of spherical gear pair, a mathematical model of arc tooth surface of hemispherical bevel gear is established, and the mathematical description of the tooth profile of spherical gear is completed by combining the equation of ring tooth surface. It provides the basis and reference for the meshing design of ball gear.
\end{abstract}

\section{Introduction}

At present, the multi degree of freedom gear is only structural design, and there is no meshing analysis and mathematical description.

The meshing motion of three degree of freedom involute spherical gear pair constructed in this paper is divided into the meshing motion of involute ring gear and the transmission between hemispherical bevel gear. The meshing motion between the involute ring tooth spherical gear is equivalent to the rolling between two spherical meridians, and the transmission between the hemispherical bevel gear is equivalent to the rolling between two spherical weft lines. The two kinds of tooth surfaces are combined to form the tooth profile of the spherical gear, so as to realize the transmission of three degrees of freedom motion. The mathematical models of the arc tooth surface of the hemispherical bevel gear and the ring tooth surface of the involute ring gear are established respectively. The data coordinates of the two kinds of tooth surfaces are obtained and imported into MATLAB software for rapid entity modeling.

\section{Analysis on meshing principle of spherical gear}

The tooth profile characteristics of existing spherical gears are summarized. As shown in Fig. 21, the meshing transmission of the spherical gear pair is equivalent to pure rolling between a pair of pitch balls, and the meridians and wefts constituting the nodal ball surface are regarded as pitch circles. The spherical gears with three degrees of freedom have meshing points on their pitch ball longitude and latitude lines. In order to realize continuous meshing without transmission principle error, the meshing of spherical gear tooth surface should be equal to the rolling of pitch circle weft or pitch circle longitude. That is to say, when the annular tooth surface is engaged in meshing, it is equivalent to rolling between pitch ball meridians, and when arc-shaped tooth surface is engaged in meshing, it is equivalent to rolling between pitch ball and weft lines.

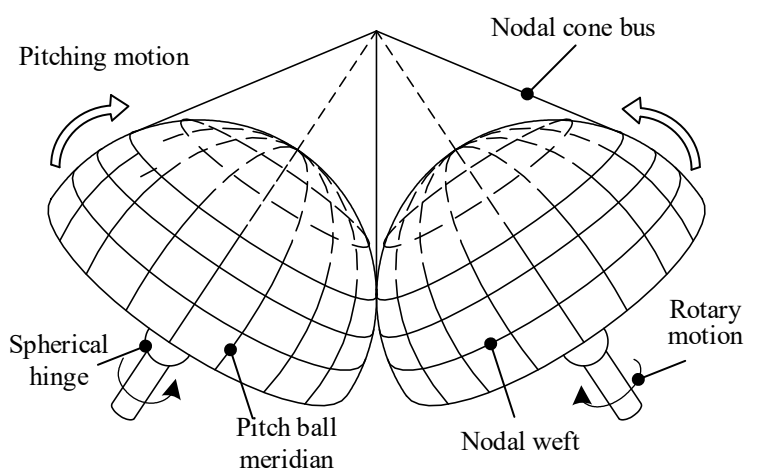

Figure. 2.1 Meshing transmission of spherical gear pair

\footnotetext{
*Corresponding author's e-mail: 635377166@qq.com
} 


\section{Mathematical model of ball gear meshing}

\subsection{Kinematics analysis of hemispherical bevel gear pair}

Hemispherical bevel gear pair can realize the transmission under the condition of variable intersecting shaft angle, only the spherical involute of arc tooth surface participates in meshing. Considering the meshing of arc tooth surface under the angle of any intersecting axis, two motion parameters need to be defined in the process of motion. As shown in Fig. 3.1, the meshing motion of a pair of hemispherical bevel gears is shown. The radius of the two segments is the same, and the $Z_{10}$-axis and $Z_{20}$-axis of the two segments coincide at the initial moment. The coordinate system $F_{1}\left(x_{1}, y_{1}, z_{1}\right)$ and $F_{2}\left(x_{2}, y_{2}, z_{2}\right)$ are fixedly connected with the driving wheel 1 and the driven wheel 2 respectively, and the distance between the two ball centers is constant. The origin $O_{10}$ and $O_{20}$ of the fixed systems $F_{10}\left(x_{10}, y_{10}, z_{10}\right)$ and $F_{20}\left(x_{20}, y_{20}, z_{20}\right)$ are the same as those of the coordinate systems $F_{1}$ and $F_{2}$, respectively.

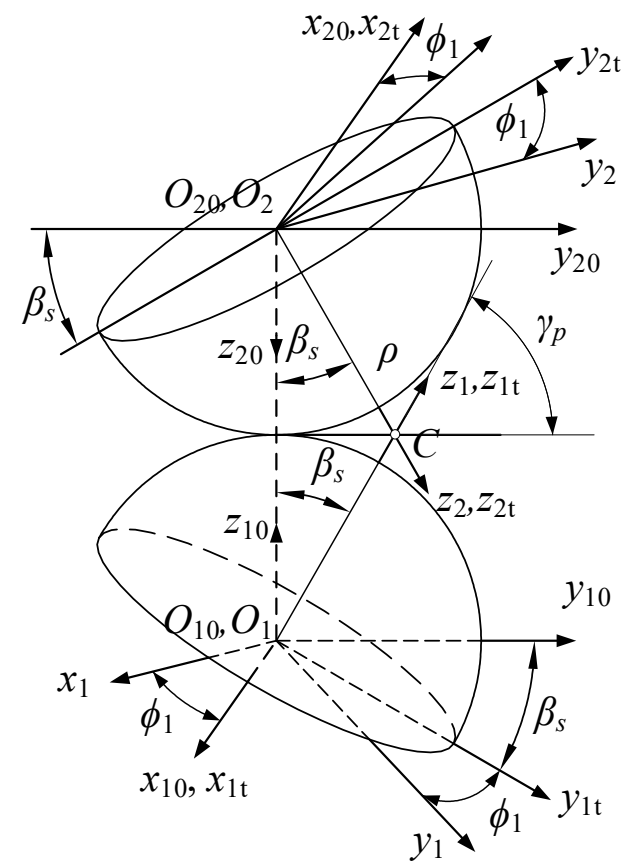

Figure. 3.1 meshing motion of hemispherical bevel gear pair

When the driving wheel 1 rotates clockwise $\beta$ around the $x_{10}$ axis from the initial position, the driven wheel 2 rotates $\beta_{s}$ clockwise around the $x_{20}$ axis from the initial position, and the arrival position coincides with the temporary coordinate system $F_{1 t}\left(x_{1 t}, y_{1 t}, z_{1 t}\right)$ and $F_{2 t}\left(x_{2 t}, y_{2 t}, z_{2 t}\right)$ respectively. This motion is the pitch motion of the spherical gear pair, which is realized by the meshing motion between the annular tooth surfaces; then, the main driving wheel 1 is turned clockwise around the $z_{1 t}$ axis $\emptyset_{1}$, Then, the driven wheel 2 rotates clockwise $\emptyset_{1}$ around the $z_{2 t}$ axis, and the arrival position coincides with $F_{1}\left(x_{1}, y_{1}, z_{1}\right)$ and $F_{2}\left(x_{2}, y_{2}, z_{2}\right)$ respectively. The transmission of the rotary motion is realized by the meshing between the arc tooth surfaces.

Fig. 3.1 the movement process of gear pair, the transformation matrix of coordinate system is established as follows:

$$
\boldsymbol{M}_{101}=\boldsymbol{M}_{101 t} \cdot \boldsymbol{M}_{1 t 1}=\left[\begin{array}{cccc}
1 & 0 & 0 & 0 \\
0 & \cos \beta_{s} & \sin \beta_{s} & 0 \\
0 & -\sin \beta_{s} & \cos \beta_{s} & 0 \\
0 & 0 & 0 & 1
\end{array}\right]\left[\begin{array}{cccc}
\cos \phi_{1} & \sin \phi_{1} & 0 & 0 \\
-\sin \phi_{1} & \cos \phi_{1} & 0 & 0 \\
0 & 0 & 1 & 0 \\
0 & 0 & 0 & 1
\end{array}\right]
$$

Where $M_{101}$ is the transformation matrix from coordinate system $F_{1}$ to fixed system $F_{10}$.

Similarly, the coordinate transformation matrix from the fixed system $F_{10}$ to the coordinate system $F_{2}$ is as follows:

$$
\boldsymbol{M}_{210}=\boldsymbol{M}_{22 t} \cdot \boldsymbol{M}_{2 t 10}=\left[\begin{array}{cccc}
\cos \phi_{1} & \sin \phi_{1} & 0 & 0 \\
-\sin \phi_{1} & \cos \phi_{1} & 0 & 0 \\
0 & 0 & 1 & 0 \\
0 & 0 & 0 & 1
\end{array}\right]\left[\begin{array}{cccc}
-1 & 0 & 0 & 0 \\
0 & \cos \beta_{s} & \sin \beta_{s} & -2 r \sin \beta_{s} \\
0 & \sin \beta_{s} & -\cos \beta_{s} & 2 r \cos \beta_{s} \\
0 & 0 & 0 & 1
\end{array}\right]
$$

According to the above derivation, the transformation matrix from driven system $F_{1}$ to dynamic system $F_{2}$ can be obtained as follows:

$$
\boldsymbol{M}_{21}=\boldsymbol{M}_{210} \cdot \boldsymbol{M}_{101}
$$




\subsection{Mathematical description of hemispherical bevel gear}

\subsubsection{Mathematical description of ring tooth surface and arc tooth surface}

The annular tooth surface is formed by the middle convex type or concave type plane involute rotating around the $Z_{10}$ axis of Fig. 2.1. The middle convex type is shown in Fig. 3.2, which is also the sectional view of the annular tooth surface of Fig. 2.1 in $x_{10} O_{10} Z_{10}$ coordinate plane. The pitch ball meridian is the pitch circle of the plane involute. The equation of annular tooth surface is as follows:

$$
r_{10}^{(k)}=r_{b}\left[\begin{array}{c}
{[\sin (u+\eta)-u \cos (u+\eta)] \cos v} \\
{[\sin (u+\eta)-u \cos (u+\eta)] \sin v} \\
\cos (u+\eta)+u \sin (u+\eta) \\
1
\end{array}\right]
$$

Where $r_{b}$ and $u_{1}$ is the radius of base circle and the sum of development angle and pressure angle. $v$ is the angle of rotation of the plane involute around the z-axis. $\eta$ is the azimuth of the latitude where the starting point of the plane involute is located. For the middle convex plane involute tooth profile, the azimuth angles of the starting points of the left and right side plane involutes are respectively $\beta_{1}(k)$ and $\beta_{1}(k)+\theta_{v}$ 。

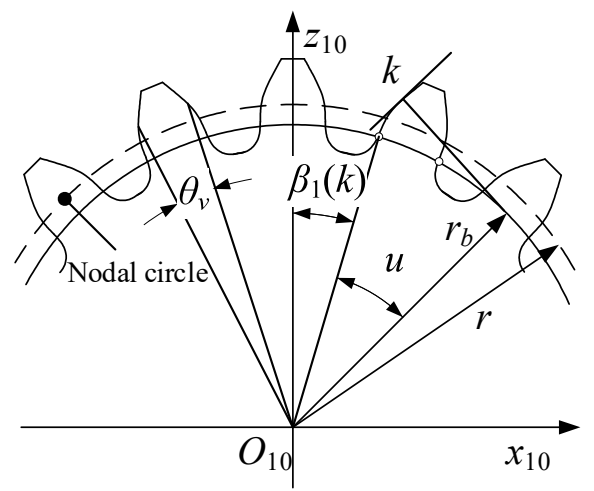

Figure. 3.2 involute ring tooth plane involute tooth profile

The parameter equation of spherical involute is as follows:

$$
\rho\left[\begin{array}{c}
\boldsymbol{r}_{s}^{(p)}=\boldsymbol{M}_{s 0} \cdot \boldsymbol{r}_{0}^{(p)}= \\
\cos \psi \sin \varphi-\sin \psi \cos \varphi \sin \gamma_{b} \\
\sin \psi \sin \varphi+\cos \psi \cos \varphi \sin \gamma_{b} \\
\cos \varphi \cos \gamma_{b} \\
1
\end{array}\right]
$$

Where, $\boldsymbol{M}_{s 0}$ is the coordinate system $F_{0}\left(x_{0}, y_{0}, z_{0}\right)$ to the coordinate system $S_{s}$, where $\rho$ is the generatrix length of the base cone and is also the bus length of the nodal cone, $\gamma_{b}$ is the base cone angle. $\psi$ is the roll angle of spherical involute, $O_{s} p$ and $O_{s} T$ The included angle of $\varphi$ is the rolling angle of circular plane, and $\varphi=$ $\psi \sin \gamma_{b}$ 。

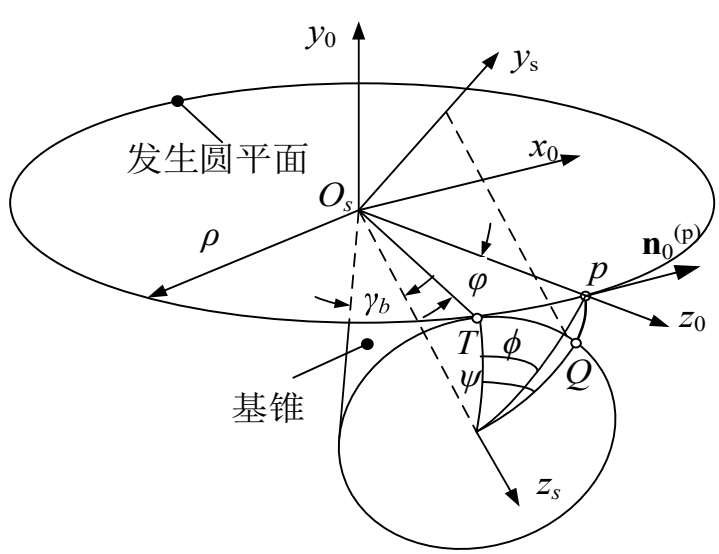

Figure. 3.3 formation of spherical involute

The unit normal vector of spherical involute is represented as $n_{0}^{(p)}=[1,0,0,0]^{T}$ in the coordinate system $S_{0}$ in Fig. 3.3, and the normal vector is free vector, so the last element of the vector is 0 . According to the coordinate transformation method, the normal vector of arc tooth surface is:

$$
\boldsymbol{n}^{(s)}=\boldsymbol{M}_{s 0} \cdot \boldsymbol{n}_{0}^{(p)}=\left[\begin{array}{c}
\cos \varphi \cos \psi+\sin \gamma_{b} \sin \varphi \sin \psi \\
\cos \varphi \sin \psi-\sin \gamma_{b} \sin \varphi \cos \psi \\
-\cos \gamma_{b} \sin \varphi \\
0
\end{array}\right]
$$




\subsubsection{Mathematical description of conjugate tooth surface of hemispherical bevel gear}

The independent variables of arc tooth surface $\Sigma^{(1)}$ are $\beta_{s}$ and $\varphi$. According to formula (3-1), conjugate tooth surface $\Sigma^{(2)}$ of arc tooth surface is expressed as follows:

$$
\boldsymbol{r}^{(1)}\left(\beta_{s}, \varphi\right)=\left[\begin{array}{cccc}
1 & 0 & 0 & 0 \\
0 & -1 & 0 & 0 \\
0 & 0 & -1 & L \\
0 & 0 & 0 & 1
\end{array}\right] \boldsymbol{r}_{s}^{(p)}=\left[\begin{array}{c}
\rho\left(\cos \eta \sin \varphi-\sin \eta \cos \varphi \sin \gamma_{b}\right) \\
-\rho\left(\sin \eta \sin \varphi+\cos \eta \cos \varphi \sin \gamma_{b}\right) \\
-\rho \cos \varphi \cos \gamma_{b}+L \\
1
\end{array}\right]
$$

Where $\rho$ is the length of nodal cone generatrix $O_{1} C$ or $\mathrm{O}_{2} \mathrm{C}$ in the above figure, 1 is the distance from the intersection point $C$ of the axis of rotation to the axis $Z_{10}$ or $Z_{20}$, and the other variables are the same as the

$$
\boldsymbol{n}^{(1)}=\left[\begin{array}{cccc}
1 & 0 & 0 & 0 \\
0 & -1 & 0 & 0 \\
0 & 0 & -1 & L \\
0 & 0 & 0 & 1
\end{array}\right] \boldsymbol{n}_{s}^{(p)}=\left[\begin{array}{c}
\cos \varphi \cos \eta+\sin \gamma_{b} \sin \varphi \sin \eta \\
\cos \eta \sin \gamma_{b} \sin \varphi-\cos \varphi \sin \eta \\
\cos \gamma_{b} \sin \eta \\
0
\end{array}\right]
$$

That is, in the coordinate system $F_{10}$, the angular velocity of driving wheel 1 and driven wheel 2 are respectively:

\subsection{Meshing equation of hemispherical bevel gear}

parameters of the spherical involute equation.

According to the coordinate transformation method, the normal vector of $\Sigma^{(1)}$ of driving gear face is obtained as follows:

The meshing equation is derived by kinematic method,

$$
\boldsymbol{\omega}^{(1)}=\left[0,-\omega \sin \beta_{s},-\omega \cos \beta_{s}\right]^{T}, \boldsymbol{\omega}^{(2)}=\left[0, \omega \sin \beta_{s},-\omega \cos \beta_{s}\right]^{T}
$$

Where $\omega$ is the modulus of the angular velocity vector. The relative speed of the gear pair at the contact point is

$$
\begin{aligned}
\boldsymbol{V}^{(12)} & =\boldsymbol{\omega}^{(1)} \times \boldsymbol{r}_{10}^{(1)}-\left(\boldsymbol{\omega}^{(2)} \times \boldsymbol{r}_{10}^{(1)}+\boldsymbol{\xi}\right) \\
& =\left|\begin{array}{ccc}
\boldsymbol{i}_{10} & \boldsymbol{j}_{10} & \boldsymbol{k}_{10} \\
0 & -2 \omega \sin \beta_{s} & -0 \\
x_{10} & y_{10} & z_{10}
\end{array}\right|-2 r \boldsymbol{k}_{10} \\
& =2\left(r-z_{0}\right) \omega \boldsymbol{i}_{10}+2 x_{10} \sin \beta_{s} \boldsymbol{k}_{10}
\end{aligned}
$$

Where $r_{10}^{(1)}$ is the vector of the meshing point on the arc tooth surface $\Sigma^{(1)}$ under the fixed system $F_{10}$, and $r_{10}^{(1)}=\left[x_{10}, y_{10}, z_{10}\right]^{T} ; \xi$ is the radial diameter from the

$$
f\left(\beta_{s}, \phi_{1}\right)=\mathbf{n}_{10}^{(1)} \cdot \mathbf{V}^{(12)}=\rho\left(-\cos \beta_{s} \cos \gamma_{b} \cos \left(\phi_{1}+\eta\right)+\sin \gamma_{b} \sin \beta_{s}\right)
$$

Where $n_{10}^{(1)}$ is the normal vector of the arc tooth surface under the fixed system $F_{10}$.

According to formula (3-7), the normal vector of driving gear tooth surface $\Sigma^{(1)}$ obtained by coordinate transformation method in fixed system $F_{10}$ is expressed as:

$$
\mathbf{n}_{10}^{(1)}=\mathbf{M}_{101} \cdot \mathbf{n}^{(1)}
$$

In this formula, since the normal vector is a free vector, the result of the above formula can be taken as the multiplication of the first three rows and three columns of $\mathbf{M}_{101}$ and the first three vector elements of $n^{(1)}$ respectively. In combination with equation $\cos \phi=$ coordinate origin $O_{10}$ to $O_{20}$, and $\xi=2 r k_{10} ; r$ is the nodal ball radius. In the coordinate system $F_{10}$, then $r_{10}^{(1)}$ is calculated as follows:

$$
\boldsymbol{r}_{10}^{(1)}=\boldsymbol{M}_{101} \cdot \boldsymbol{r}^{(1)}
$$

The hemispherical bevel gear pair satisfies the spatial meshing principle, and the pair of tooth surfaces $\Sigma^{(1)}$ and $\Sigma^{(2)}$ must be tangent at the contact point, that is, the normal vectors of the two gear surfaces at the contact point coincide. In order to ensure the continuous meshing transmission of the two tooth surfaces, the relative velocity at the contact point has no component in the normal direction of the common normal. Therefore, according to the above formulas, the meshing equation is deduced by using the symbolic calculation function of Mathematica

$\frac{\tan \gamma_{b}}{\tan \gamma}$, the above meshing equation is further simplified

$$
\left\{\begin{array}{l}
\phi_{1}=\phi_{p}\left(\beta_{s}\right)-\eta \\
\phi_{p}\left(\beta_{s}\right)=\arccos \left(\tan \gamma_{b} / \tan \gamma_{p}\right)
\end{array}\right.
$$

A pair of spherical involutes are meshed as conjugate curves at any pitch angle $\beta_{s}$ position. The conjugate tooth surface $\Sigma^{(2)}$ is also composed of a family of spherical involutes with single parameter.

$$
\left\{\begin{array}{l}
\mathbf{r}^{(2)}\left(\beta_{s}, \psi, \phi_{1}\right)=\mathbf{M}_{21} \cdot \mathbf{r}^{(1)} \\
\phi_{1}=\phi_{p}\left(\beta_{s}\right)-\psi \\
\phi_{p}\left(\beta_{s}\right)=\arccos \left(\tan \gamma_{b} / \tan \gamma_{p}\right)
\end{array}\right.
$$




\section{Conjugate tooth surface simulation of hemispherical bevel gear pair}

The single degree of freedom meshing principle of hemispherical bevel gear pair is analyzed. In this section, the conjugate tooth surface is simulated by MATLAB programming to verify the derivation of meshing principle. The program flow chart is shown in Figure 3.4.

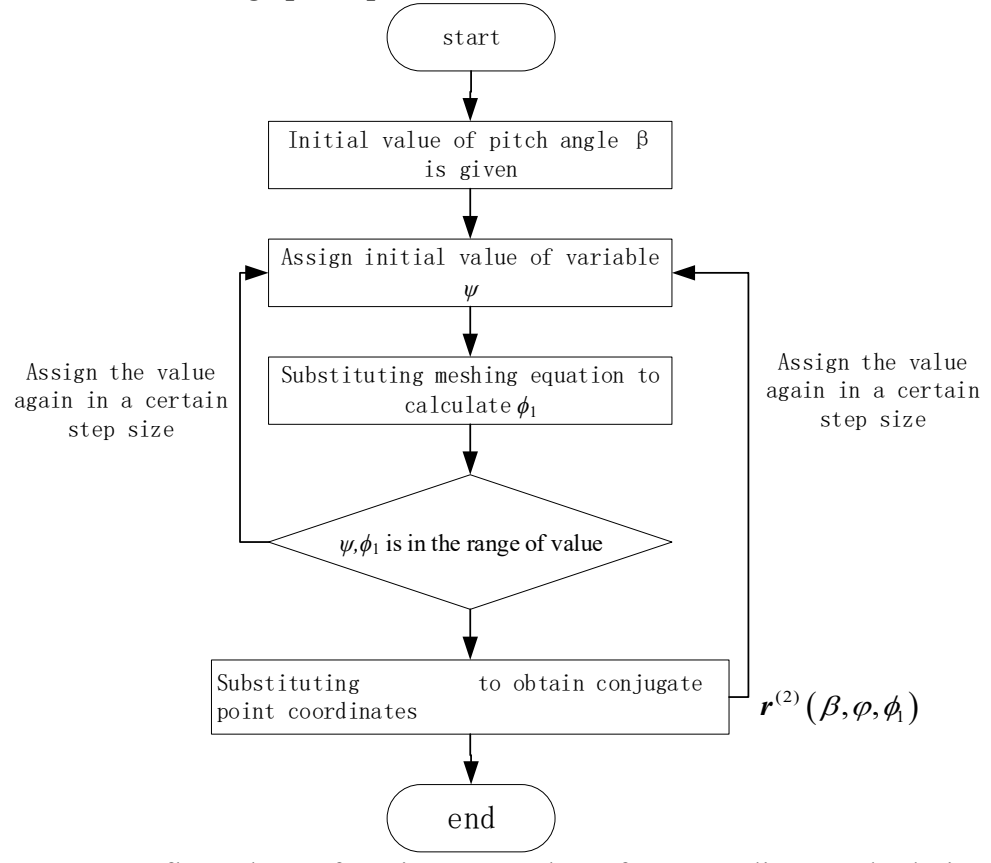

Figure 3.4 flow chart of conjugate tooth surface coordinate calculation Through MATLAB programming, the tooth surface simulation is shown in Fig. 3.5.

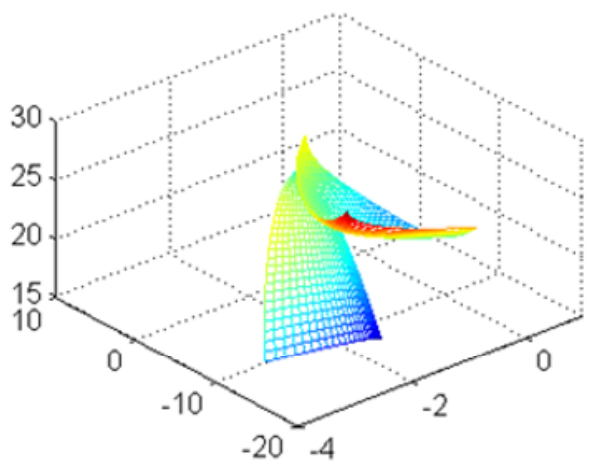

$\beta=20^{\circ}$

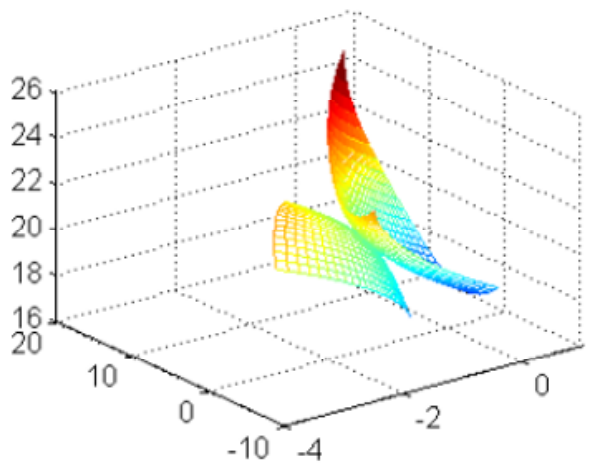

$\beta=40^{\circ}$

Figure 3.5 arc tooth surface and its conjugate tooth surface

\section{Conclusion}

According to the above equations, the two kinds of tooth surfaces are simulated by MATLAB. At the same time, it is verified that the above meshing equation (3-14) has only one set of solutions. When the angle $\beta$ of the intersecting axes is fixed, the contact point between the arc tooth surface and its conjugate tooth surface moves along the spherical involute, which verifies that the conjugate tooth surface is also a family of spherical involute curves. The meshing of hemispherical bevel gears is equivalent to the transmission between spherical involute bevel gears with infinitesimal thickness at any intersection angle.

\section{Reference}

1. Al-Daccak M J, Angeles J, González-Palacios M A. The Modeling of Bevel Gears Using the Exact Spherical Involute[J]. Journal of Mechanical Design, 1994,116(2):364-368.

2. Ting L, Cunyun P, Fudong G, et al. Research on sliding ratios of conjugate surfaces of two degrees of freedom meshing transmission of spherical gear pair[J]. Journal of Mechanical Design, 2012,134(9):91002.

3. Cun-yun P, Xi-sen W, Kun-yu Y, et al. Research on transmission principle and kinematics analysis for involute spherical gear[J]. Frontiers of Mechanical Engineering, 2006,1(2):183-193. 
4. Fuentes-Aznar A, Gonzalez-Perez I. Mathematical definition and computerized modeling of spherical involute and octoidal bevel gears generated by crown
gear[J]. Mechanism \& Machine Theory, 2016,106:94-114. 